\section{IMPROVING PATIENT CARE AND SAVING CLINICIAN TIME WITH A NEW ELECTRONIC NICU PROFORMA}

Freja Mahler, Nils Wijtzes, Sina Durani.

10.1136/bmjpo-2021-RCPCH.158

\section{Background}

Objectives Documentation is crucial for communication and patient safety. A major role of neonatal doctors during the night shift is to prepare daily ward round sheets for the next morning. We noted that this was time consuming, especially when preparing for the weekly multidisciplinary team (MDT) meeting. Moreover, patient data often was incomplete, most notably with regards to the documentation of parental communications in the first 24 hours after admission. That first encounter with parents is crucial as it usually captures the reason for admission and the projected course, including anticipated complications. Our aim, therefore, was to create a new daily ward round sheet which could address all these problems.

Methods We carried out an initial audit and two user surveys regarding the existing proforma.

1. A retrospective audit of the notes of 13 babies recently admitted to the neonatal ITU. We examined completion of the information recorded in the first 24 hours of life including parental communication.

2. A survey looking at time spent during the night by doctors filling in the existing proforma.

3. An opinion survey on the current proforma.

The results were presented at a departmental teaching session. Incorporating peer feedback, we created a new proforma using excel. Peers were trained to use the new proforma, which was instated for a period of three weeks. An initial follow up questionnaire was sent, focusing on any potential and immediate safety concerns.

Results The initial survey revealed that the mean time to complete the existing proforma was 31 minutes during normal nights and 158 minutes on the night prior to the MDT. Our initial opinion survey showed a varied response to the question whether filling in time during a regular night shift was acceptable. A majority, however, thought it took too long to complete the existing proforma on the night shift prior to the MDT.

Moreover our audit demonstrated that most babies had key information missing, for instance hospital number was documented in $62 \%$ of the cases and in only $23 \%$ of the cases the proforma was signed. Parental communication was recorded in $23 \%$ of the cases. Following the introduction of the new proforma, $86 \%$ of doctors felt the new proforma was better, $14 \%$ did not have a preference $(n=13)$. Most of the feedback stated that it saved time and made information easier to read. $29 \%$ of respondents identified potential safety concerns mainly regarding team members copying and not checking information accuracy. No immediate safety concerns were raised.

Conclusions The initial feedback on the new proforma shows that it is more user friendly, saves time and only has minor flaws. We are in the process of re-surveying time taken to fill in the new proforma as well as re-auditing the recording of important information and parental communication. Following this, we will adjust the new proforma and start the next PDSA cycle.

\section{ONLINE LISTENING AND SHARING SESSIONS AS A TOOL TO AID RAPID INTERNATIONAL KNOWLEDGE TRANSFER DURING THE COVID-19 PANDEMIC}

Jenny Carroll, Alicia Spittle, Gillian Saloojee, Johannes Verheijden, Laura Yeo, Guorong Wei. UK

\subsection{6/bmjpo-2021-RCPCH.159}

Background The global Covid-19 pandemic disrupted services for children with disabilities and their families, leading families, clinicians, and researchers working in the field of childhood disability, to rapidly re-evaluate and adapt interventions. To understand the impact of Covid-19 on children and families across the globe, and assist clinicians around the world in adapting and adjusting to new ways of delivering services which would continue to meet families' need in local contexts, the Global Professional Education Committee of the International Alliance of Academies of Childhood Disability (IAACD) established a Covid-19 Task force. The task force comprised three subgroups, one of which was responsible for collecting and disseminating relevant service innovation and information via webinars. The subgroup represented 7 academies and 14 countries.

\section{Objectives}

- To understand the impact of COVID-19 on service delivery from clinicians', researchers', and families' perspectives

- To provide a mechanism for rapid sharing of useful multidisciplinary knowledge and practice across the globe

- To understand how shared learning could be adapted to local contexts

- To build a global network of professionals supporting children with disability able to rapidly share knowledge and practice

Methods Recognising that there was no experience or established best practice in a pandemic, the group decided the appropriate mechanism for sharing and collating knowledge and experience was the development of a Listening and Sharing platform, rather than merely disseminating knowledge via webinars. The group produced guidelines on how member countries/academies could organise online Listening and Sharing sessions for their regions. These were shared on the IAACD website. Several online Listening and Sharing sessions took place around the world culminating in a 24-hour global Listening and Sharing session on World CP day 2020. Sessions from this 24-hour event were recorded and posted on the IAACD website with open access to facilitate further sharing and to create a freely available resource.

Results

- The work of the Listening and Sharing subgroup culminated in

\section{Abstract 285 Table 1}

Question-Satisfaction with: Responses (multiple choice-Excellent, Satisfactory, Neutral, Bit disappointing, Poor)

Session format Excellent(71\%) Satisfactory(24\%) Neutral(5\%)

Overall content Excellent $(71 \%)$ Satisfactory $24 \%$ ) Neutral(5\%)

Content relevance of $(5=$ most $5(57 \%) 4(29 \%) 3(5 \%) 3(5 \%) 1(5 \%)$

relevant=1 least) 